\title{
Self-help Interventions for Anxiety Disorders: An Overview
}

\author{
Pim Cuijpers, PhD, and Josien Schuurmans, PhD
}

\author{
Corresponding author \\ Pim Cuijpers, PhD \\ Department of Clinical Psychology, Vrije Universiteit Amsterdam, \\ Van der Boechorststraat 1, 1081 BT, Amsterdam, The Netherlands. \\ E-mail: P.Cuijpers@psy.vu.nl
}

Current Psychiatry Reports 2007, 9:284-290

Current Medicine Group LLC ISSN 1523-3812

Copyright (C) 2007 by Current Medicine Group LLC

Anxiety disorders are highly prevalent and are associated with a marked impairment in quality of life and a huge economic cost to society. Unfortunately, a considerable number of people who struggle with anxiety do not seek or receive adequate treatment. Self-help interventions have been proposed to constitute a relatively cheap, effective, efficient, and low-threshold intervention for anxiety disorders. This paper offers a critical discussion of their advantages and disadvantages and the evidence for their effectiveness. We conclude that guided self-help can play a major role in mental health care for patients with anxiety disorders. However, several research questions need to be answered before broad-scale dissemination is possible. The Internet will continue to play a prominent role in the further development of this field of research and clinical practice.

\section{Introduction}

Anxiety disorders are highly prevalent, affecting approximately one in six people during their lifetime and one in eight during 1 year [1,2]. These disorders are associated with a marked impairment in the quality of life of patients and their relatives [3,4] and incur huge economic costs [5]. A Dutch population study estimated the cost of anxiety disorders in a population of 1 million to be approximately $\$ 500$ million per year [6].

Despite the availability of effective psychological and pharmacologic treatments for anxiety disorders, a considerable number of people suffering from anxiety do not seek any professional help [7,8]. Furthermore, those who do seek treatment are frequently confronted with long waiting periods and often are not provided with evidencebased treatments [9]. Thus, it is important to develop evidence-based and cost effective treatments that are eas- ily accessible for patients and that take up as little time of therapists and researchers as possible [10•].

Self-help interventions to constitute such an intervention have been proposed. In this paper, we will address the nature and different types of self-help interventions and will provide a critical discussion of the available evidence for their effectiveness and their advantages and disadvantages.

\section{What is a Self-help Intervention?}

A self-help intervention can be defined as a psychological treatment in which the patient takes home a standardized psychological treatment protocol and works through it more or less independently. This protocol involves a guide that describes the steps that the patient can take in order to apply a generally accepted psychological treatment to himself or herself. The standardized psychological treatment protocol can be written down in book form, but it also can be available through other media, such as a personal computer, CD-ROM, television, video, or the Internet. Contacts with therapists are not necessary for the patient to complete the therapy. If the protocol does entail contacts with a therapist, these contacts are only supportive or facilitative in nature and are not aimed at developing a traditional relationship between therapist and patient. Instead, any contact is aimed at providing support and, if necessary, added explanation for working through the standardized psychological treatment. Contacts with therapists can be provided through personal contact, by telephone, by e-mail, or by any other available means of communication.

Other terms that have been used for self-help interventions include minimal contact psychotherapy [11], bibliotherapy $[12,13]$ and self-administered psychotherapy or treatment [14-16]. Self-help interventions have been developed for a variety of disorders and problems, including problem drinking, anxieties, lack of social skills, migraine, eating disorders, smoking, sexual dysfunction, and insomnia $[13,17,18]$. Most self-help interventions for anxiety disorders are based on cognitive-behavioral techniques, such as exposure, cognitive restructuring, and applied relaxation. Cognitive-behavioral interventions represent current state-of-the-art treatment for anxiety disorders, 
as they have been shown to be effective and often superior to other modes of treatment for anxiety disorders in large numbers of well-designed randomized studies and metaanalyses [19,20]. A far more pragmatic reason for choosing cognitive-behavioral techniques is the fact that these techniques tend to be very straightforward and therefore can be readily broken up into relatively easy steps, as opposed to most other common psychological interventions, such as psychodynamic or interpersonal therapies.

\section{Different Types of Self-help Interventions}

Self-help interventions can be delivered in a variety of different formats and settings. Although not exhaustive, the following major types of self-help can be distinguished:

- Unguided self-help. First, many self-help books can be bought in a bookstore by anyone who thinks they may be of benefit. There is no professional or paraprofessional support, and the "patient" can discontinue "treatment" anytime he or she chooses. It also is possible to deliver unguided selfhelp through the Internet or through stand-alone personal computer programs.

- Self-help as partial replacement of face-to-face therapy. Self-help interventions also can be used as part of a regular treatment. For example, a therapist providing face-to-face treatment can give a patient a self-help book in order to speed up the treatment process or to give the patient the opportunity to learn the principles of the therapy outside therapy. The patient also could receive a palmtop computer as part of a regular treatment, in which the palmtop is used to prompt the patient to practice one of the therapy components. It also often happens that a therapist advises the patient to buy a self-help book on a related problem, such as a sleep problem or a mild alcohol problem that is not the focus of the therapy but does interfere with the patient's functioning.

- Self-help as an independent intervention. Self-help can be delivered as an independent intervention. Usually, there is some support from a professional or paraprofessional in this type of intervention. Many studies that have focused on the effectiveness and efficacy of self-help interventions have chosen this format. In these studies, the patient receives a self-help book and works through it independently while a professional or paraprofessional has contact with the patient by telephone at regular times. These contacts are brief and are not aimed at developing a traditional relationship between patient and therapist. Instead, contacts are aimed primarily at providing added explanation about the method when necessary and at supporting and stimulating the patient to continue the treatment. In recent years, the Internet has grown more and more important as a medium for delivering guided and independent self-help treatments. We will describe these developments in more detail subsequently.

Most research has focused on this third type of selfhelp, as it constitutes an independent minimal-contact intervention that might be used as an alternative to psychotherapy or medication. Therefore, our discussion of the usefulness of self-help for the treatment of anxiety will focus primarily on this type of intervention.

\section{Common Components of Self-help Interventions for Anxiety Disorders}

Although the exact contents of self-help interventions differ for each intervention, several components are used in most interventions:

- Psychoeducation. Information on anxiety disorders, what they are, what causes them, the vicious cycle that perpetuates anxiety, and what can be done about it is part of most self-help interventions.

- Relaxation. Although the effects of relaxation techniques on anxiety probably are not very strong, except in generalized anxiety disorder, the opportunity to learn these techniques is very much appreciated by many patients. Several systematic relaxation techniques are available, with applied relaxation (originally developed by Öst [21] in 1986) being the most widely used. With these techniques, the patient learns to relax his or her body systematically and then to relax while entering anxious situations.

- Graded exposure. The principle of exposure is that the patient makes a hierarchy of situations that are increasingly anxious. The patient enters into the situation that is somewhat frightening and remains there until the anxiety diminishes and disappears. Then a more frightening situation is chosen and the procedure is repeated until the anxiety has disappeared completely. Exposure has been proven to be highly effective in all phobias (agoraphobia, social phobia, and specific phobias) and also is often incorporated into self-help for obsessivecompulsive disorder.

- Cognitive restructuring. The basic idea of cognitive restructuring is that anxiety disorders are caused by dysfunctional cognitions. In cognitive restructuring, the patient tries to discover which dysfunctional cognitions related to anxiety he or she has. Then the patient registers in which situations the cognitions exist and how they can be 
challenged and changed in such a way that they do not stimulate the anxiety.

- Anxiety management and other techniques. Several other smaller techniques often are used in self-help approaches, including positive self-talk, imaginary exposure, contraconditioning, and systematic positive stimulation when an assignment has been conducted. These smaller techniques often are presented as anxiety management together with elements of cognitive restructuring, relaxation, and exposure.

\section{Effects of Self-help Interventions on Anxiety Disorders}

The first randomized controlled studies examining the effects of self-help interventions on anxiety disorders date from the late 1960s and early 1970s [22,23]. Since then, several dozen controlled and comparative studies have been conducted. In the most recent and largest metaanalysis, conducted by Hirai and Clum [10•], 33 studies were included. The standardized effect size (Cohen's d) as compared with wait list conditions was 0.68 (95\% CI = $0.57 \sim 0.79$ ), indicating a moderate to large effect. This finding is in concordance with findings from earlier metaanalyses [24,25]. The meta-analysis by Hirai and Clum [10 $]$ also examined different characteristics of the studies and whether these were related to the effect size. No indication was found that the effect size was related to type of anxiety disorder, although most research was aimed at panic, social anxiety disorder, and phobias, whereas few studies examined patients with generalized anxiety disorder and post-traumatic stress disorder. Furthermore, no differences in effect sizes were found among clinical, community, and college student samples; for studies in which a diagnostic interview was used to determine the presence of an anxiety disorder compared with studies in which only self-report measures were used; between studies that provided professional or paraprofessional support versus unguided self-help; or for studies in which written materials were used compared with studies in which audio/video or computerized materials were used. Only one factor was found to be related to effect size: estimates of effect size were larger when self-report measures were used (mean $\mathrm{d}=0.75 ; 95 \% \mathrm{CI}=0.63 \sim 0.87$ ) as compared with clinician-rated measures $($ mean $\mathrm{d}=0.17$; $95 \% \mathrm{CI}=$ $0.03 \sim 0.31$ ).

With regard to relative effectiveness, this meta-analysis showed that self-help treatments were less effective than face-to-face treatments (standardized difference for all studies was $\mathrm{d}=-0.42 ; 95 \% \mathrm{CI}=-0.62 \sim-0.22$ ). However, this difference was only significant for studies in which unguided self-help was provided $(\mathrm{d}=-0.62 ; 95 \%$ $\mathrm{CI}=-0.86 \sim-0.49$ ). Studies in which regular support was given during the self-help treatment did not significantly differ from face-to-face therapies $(\mathrm{d}=-0.11 ; 95 \% \mathrm{CI}=$
$-0.42 \sim-0.20)$. The mean effect size for this type of selfhelp intervention was $0.68(95 \% \mathrm{CI}=0.54 \sim 0.83)$.

Internet-based Self-help for Anxiety Disorders In recent years, more and more self-help treatments have become available through the Internet. Since 2001, a considerable number of randomized controlled and comparative trials examining the effects of Web-based self-help interventions have been published, and most of these were published after 2005 [26-31]. This rapidly growing research area reflects the possibilities of the Internet for researching and implementing Web-based self-help interventions. The Internet facilitates the implementation of self-help interventions, it provides various options to make the intervention more appealing (by adding graphics, audio, and video), it facilitates the inclusion of self-report questionnaires, and it can provide direct (automated) feedback about the results to participants. Furthermore, the Internet greatly simplifies the process of effect research, as data collection can be conducted automatically through the Internet. In the next few years, it is expected that the exponential growth in the number of trials on Internet self-help will continue. Recently, an international organization of researchers examining the effects of self-help interventions was founded [32]. In a recent meta-analysis, the effects of Internet-based selfhelp interventions for mood and anxiety symptoms were examined [33•]. Twelve studies, with a total of 2334 subjects, were included in this meta-analysis, and seven of them focused on subjects with anxiety disorders. The overall mean effect (Cohen's d) of the studies on anxiety was $0.96(95 \%$ CI $=0.69 \sim 1.22)$, which can be considered a large effect. This meta-analysis also made it clear that some form of guidance or support is very important in Web-based self-help. The mean effect size for studies in which no support was given was 0.26 (95\% CI $=0.08$ $\sim 0.44$ ), which is considered small, whereas the studies in which some form of support was given resulted in a large mean effect size of 1.00 (95\% CI $=0.75 \sim 1.24)$.

\section{Advantages of Self-help Interventions}

One obvious advantage of self-help treatments is that they may save therapist time. In the previous paragraph, we discussed findings demonstrating the effectiveness of self-help interventions. However, some form of guidance does seem necessary in order to optimize treatment effect, as unguided self-help interventions are significantly less effective than face-to-face treatments. Self-help treatment with regular support has demonstrated effectiveness, with no significant differences in effect size when compared with face-to-face treatments.

In light of these findings, it is only logical that clinicians and researchers frequently have suggested that self-help treatments should be implemented in stepped care 
approaches to the treatment of anxiety disorders [24,34]. In a stepped care approach, patients are provided with a lowcost intervention in the first steps, with more intensive and costly interventions reserved for those insufficiently helped by the initial intervention [8]. Stepped care models represent attempts to maximize the effectiveness and efficiency of decisions about allocation of resources in therapy. Not all patients need the same type and intensity of intervention, and for some, self-help interventions (with the support of a trained paraprofessional) may be sufficiently successful in reducing anxiety. When self-help proves to be insufficiently effective for a particular patient, a more intensive treatment can be offered, such as a standardized individual treatment by a highly trained professional therapist $[35,36]$. Although guided self-help interventions undoubtedly are not successful for every patient, the available research findings suggest that there are many patients for whom self-help interventions are sufficient to treat their problems successfully. Therefore, self-help interventions seem to be a good candidate for a first step in a stepped care intervention for anxiety disorders. If a self-help intervention does not result in satisfactory relief of anxiety, a more intensive intervention such as a face-to-face or pharmacologic treatment can be advised.

Another advantage of self-help interventions is that it may be possible to reach populations with anxiety disorders who cannot be reached with other, more traditional forms of treatment. Population-based research has shown that approximately $60 \%$ of patients with anxiety disorders do not receive any treatment at all [7,37]. There are several reasons why patients with anxiety disorders do not seek professional help [38], including fear of stigma, prejudices about therapists, lack of willingness to talk to strangers about personal problems, or physical obstacles such as walking problems or long distances. Help-seeking in patients suffering from anxiety also may be complicated by disorder-related aspects. Anxiety disorders tend to be characterized by high levels of avoidance of those situations that provoke anxiety. Treatment almost inevitably entails a confrontation with (an aspect of) the feared stimulus. For example, patients with social anxiety disorder often are reluctant to seek help because avoiding contact with other people is part of their disorder. In summary, as a large group of people with anxiety for whatever reason seem reluctant to seek professional help for their problems in a common mental health care setting, it is highly likely that a subgroup of patients actually prefer to follow a less stigmatizing and low-threshold self-help-based intervention. Another reason patients do not receive professional help is that professionals, such as general practitioners, often do not recognize the disorder or have had negative experiences with mental health care settings in the past (eg, extensive waiting periods for treatment) and therefore are reluctant to refer patients to mental health care.

It may be possible to reach a segment of this population with self-help interventions. Internet-based interventions are especially interesting for this population, as contact with professionals and researchers often can be kept to a minimum. Several studies have shown that Internet-based treatments are very effective in the treatment of anxiety disorders $[33 \bullet, 39-42]$.

Self-help therapies may have several other advantages. They may reduce waiting lists, allow patients to work at their own pace, abolish the need to schedule appointments with a therapist, save traveling time, reduce the stigma of going to a therapist, and ease help for the hard of hearing, as self-help treatments typically work more with visual than auditory information [43]. When self-help is delivered through a personal computer, it has several more advantages. For example, computer-guided self-help can quickly and automatically report patient progress and self-ratings and may be programmed to enhance patients' motivation by presenting a wide range of attractive audiovisual information with voices giving instructions in whichever gender, age, accent, language, and perhaps game format the client prefers [43].

\section{Disadvantages and Dangers}

The evidence presented so far speaks for the immediate and widespread implementation of guided self-help interventions for anxiety disorders. However, there are always two sides to any coin, and self-help treatments are no exception. This paragraph points out disadvantages and dangers to the implementation of self-help treatments. One important danger involves the lack of appropriate diagnostic procedures when patients start working with a self-help book (or Internet program) without the involvement of a professional therapist. Without proper diagnosis, patients may start with a self-help treatment targeted at a particular mental disorder despite the fact that they actually suffer from another mental disorder or possibly do not suffer from any mental disorder whatsoever. Anxiety symptoms may be transitory and a normal part of reacting to life's stresses. More importantly, anxiety symptoms can be produced by somatic causes such as hyperthyroidism. Therefore, people may mistakenly involve themselves in a particular self-help intervention when another treatment may be more appropriate. Another risk is that patients do not apply the treatment in an appropriate fashion. These risks could entail a waste of time and energy for the participant, but this might also result in the aggravation rather than the amelioration of symptoms.

Another important danger of using self-help interventions (without professional guidance) is that the patient might not succeed in finishing the therapy. For example, in an early study, it was found that only $50 \%$ of those who started with a bibliotherapy program for phobia finished it [44]. A more recent study shows that open access to a website for affective disorders often results in dropout rates of greater than $50 \%$ [33•]. Although there are no indications that the dropout rates are higher in self- 
help interventions in which personal guidance is provided than in traditional psychotherapies, dropout rates are considerably higher for Internet therapy and in self-help treatments without professional involvement. However, it should be noted that the concept of dropout is difficult to define within the context of unguided self-help treatments and open-access websites. In fact, within this context, it may constitute a completely different phenomenon than "true" dropout in traditional psychotherapy.

One could argue that working through a self-help intervention and dropping out early will not do any harm to the patient. However, it is possible that this will have negative effects. For example, not succeeding in working through the intervention may be another disappointment that strengthens feelings of helplessness and a lack of mastery, which in turn may exacerbate symptoms of depression and anxiety. Furthermore, it may be possible that a patient dropping out of a self-help cognitive-behavioral intervention will then be reluctant to accept a face-to-face cognitive-behavioral intervention because it is based on the same principles as the therapy that did not work for him or her.

Computer-aided self-help interventions may have other disadvantages [43]. Technophobia may prevent patients from using them: no computer program is equipped to answer all possible questions users may ask, it can't detect subtle nonverbal and verbal clues to clients' misunderstandings, it may stimulate clients to cherry-pick from a range of homework options presented, and not all clients regard computer interventions as acceptable [43].

Whether self-help has negative consequences is an empirical question, and as far as we know, this has not been examined in empirical research. Before definite conclusions can be drawn, we need research to examine these questions. However, in light of the current state of knowledge, it does seem clear that guided self-help in which baseline symptomatology and progress during treatment are well monitored should be preferred over unguided self-help. Also, in light of the potentially harmful effects described in this section, it should be advised that self-help books and unguided self-help websites offer a straightforward account of the dangers of early dropout and guidelines for those who do choose to discontinue the self-help intervention.

\section{Discussion and Future Directions}

In light of the available evidence, there is no doubt that guided self-help can play a major role in mental health care for patients with anxiety disorders. There is ample research showing that it is effective and that it is feasible and acceptable to patients. The Internet will play a major role in the further development and research of guided self-help in this area and definitely will speed up the developments.

However, there are several research questions that have to be answered before broad-scale dissemination is possible. Very little is known about the patients who may benefit from self-help. It is clear that many patients consider self-help interventions (through a book, through the Internet, or through other media) to be an acceptable alternative to face-to-face treatments. On the other hand, it seems obvious that there also are many patients for whom self-help is not an acceptable alternative to more traditional treatments. However, we lack the knowledge to appropriately distinguish these two groups of patients.

Another issue that has yet to be properly addressed is the amount of therapist time needed to optimize self-help interventions. Research in this area has clearly shown that no guidance results in considerably smaller effects than traditional psychotherapy and that guided self-help is as effective as face-to-face treatments. However, this does not answer the question as to the minimal amount of therapist time needed for a self-help intervention to be effective.

A similar topic is the amount of experience and expertise needed to optimize guided self-help interventions. Although a direct comparison between guidance by psychotherapists and guidance by paraprofessionals or students has not been made in a randomized controlled trial, self-help is coached by students and preregistration nurses in several studies, and results suggest that the amount of experience of those delivering or coaching the treatment does not constitute a decisive factor in treatment effectiveness (eg, [43]).

A topic of concern that is often voiced by professional therapists is the possible replacement of existing trained psychotherapists by simple self-help protocols, for if selfhelp is as effective as traditional face-to-face treatment, why not apply it to all patients and abolish the more timeconsuming and costly face-to-face treatments altogether? However, this is a very unlikely development. First of all, the sheer number of patients with anxiety and other common mental disorders is so high that the demand for professional help probably will always exceed the potential of existent mental health care settings. Self-help treatments probably will help a large subgroup of people who otherwise would receive no treatment whatsoever for their ailment. In many countries there is a shortage of well-trained cognitive-behavioral psychotherapists, and waiting lists are not an uncommon phenomenon.

Probably the best way to implement self-help interventions in routine care is as one step in stepped care interventions $[45,46]$.

\section{Limitations}

In this article, we tried to present a comprehensive overview of self-help interventions for anxiety disorders. We did not have sufficient space to present details of self-help for specific disorders or to differentiate between interventions for patients with formal diagnoses and participants who score above the cutoff score on a self-report measure. For a comprehensive review on self-help and minimal- 
contact therapies for specific anxiety disorders, the reader may wish to consult the review by Newman et al. [47] that was published in 2003 .

Our review is also limited by the currently available evidence, with several research questions still not being answered adequately. For example, the long-term effects of self-help are not yet very clear, and it is not known who prefers to receive a (guided) self-help intervention instead of a traditional treatment and who does not. As indicated earlier, guided self-help may be best implemented through stepped care interventions. Whether this is actually a good approach is not yet very clear.

\section{Conclusions}

Self-help treatments have been developed and tested in dozens of studies over almost 40 years. This research has clearly shown that self-help for anxiety disorders is effective, feasible, and acceptable for many patients. Furthermore, self-help interventions may play an important role in providing effective treatment to a large proportion of people suffering from anxiety who are not adequately treated. It is time to start implementing self-help treatment in routine practice and to fulfill the promise of self-help treatments in reducing the disease burden of anxiety disorders in the population.

\section{Acknowledgments}

Neither author has a potential conflict of interest, financial or otherwise.

\section{References and Recommended Reading}

Papers of particular interest, published recently, have been highlighted as:

- Of importance

$\bullet \quad$ Of major importance

1. Somers JM, Goldner EM, Waraich P, Hsu L: Prevalence and incidence studies of anxiety disorders: a systematic review of the literature. Can J Psychiatry 2006, 51:100-113.

2. Bijl RV, Ravelli A, van Zessen G: Prevalence of psychiatric disorder in the general population: results of The Netherlands Mental Health Survey and Incidence Study (NEMESIS). Soc Psychiatry Psychiatr Epidemiol 1998, 33:587-595.

3. Mendlowicz MV, Stein MB: Quality of life in individuals with anxiety disorders. Am J Psychiatry 2000, 157:669-682.

4. Angermeyer MC, Kilian R, Wilms HU, Wittmund B: Quality of life of spouses of mentally ill people. Int J Soc Psychiatry 2006, 52:278-285.

5. DuPont RL, Rice DP, Miller LS, et al.: Economic costs of anxiety disorders. Anxiety 1996, 2:167-172.

6. Smit F, Cuijpers P, Oostenbrink J, et al.: Costs of nine common mental disorders: implications for curative and preventive psychiatry. J Ment Health Policy Econ 2006, 9:193-200.

7. Bijl RV, Ravelli A: Psychiatric morbidity, service use, and need for care in the general population: results of The Netherlands Mental Health Survey and Incidence Study. Am J Public Health 2000, 90:602-607.
8. Haaga DA: Introduction to the special section on stepped care models in psychotherapy. J Consult Clin Psychol 2000, 68:547-548.

9. Andrews G, Issakidis C, Sanderson K, et al.: Utilising survey data to inform public policy: comparison of the cost-effectiveness of treatment of ten mental disorders. $\mathrm{Br} J$ Psychiatry 2004, 184:526-533.

10. Hirai M, Clum GA: A meta-analytic study of self-help interventions for anxiety problems. Behav Ther 2006, 37:99-111.

The most recent and comprehensive meta-analysis of self-help interventions for anxiety showing promising results for guided self-help.

11. Willemse GR, Smit F, Cuijpers P, Tiemens BG: Minimalcontact psychotherapy for sub-threshold depression in primary care. Randomised trial. Br J Psychiatry 2004, 185:416-421.

12. Cuijpers P: Bibliotherapy in unipolar depression: a metaanalysis. J Behav Ther Exp Psychiatry 1997, 28:139-147.

13. Marrs RW: A meta-analysis of bibliotherapy studies. Am J Community Psychol 1995, 23:843-870.

14. Mains JA, Scogin FR: The effectiveness of self-administered treatments: a practice-friendly review of the research. J Clin Psychol 2003, 59:237-246.

15. Ostgood-Hynes D, Greist JH, Marks IM, et al.: Selfadministered psychotherapy for depression using a telephone-accessed computer system plus booklets: an open US-UK study. J Clin Psychiatry 1998, 59:358-365.

16. Scogin F, Floyd M, Jamison C, et al.: Negative outcomes: what is the evidence on self-administered treatments? J Consult Clin Psychol 1996, 64:1086-1089.

17. Gould RA, Clum GA: A meta-analysis of self-help treatment approaches. Clin Psychol Rev 1993, 133:169-186.

18. Scogin F, Bynum J, Stephens G, Calhoon S: Efficacy of selfadministered treatment programs: meta-analytic review. Prof Psychol Res Pr 1990, 21:42-47.

19. Emmelkamp PM: Behavior therapy with adults. In Bergin and Garfield's Handbook of Psychotherapy and Behavior Change. Edited by Lambert M. New York: Wiley; 2004:393-446.

20. Gould RA, Safren SA, Washington DO, Otto MW: A meta-analytic review of cognitive-behavioral treatments. In Generalized Anxiety Disorder: Advances in Research and Practice. Edited by Heimberg RG, Turk CL, Mennin DS. New York: Guilford Press; 2004:248-264.

21. Öst LG: Applied relaxation: description of a coping technique and review of controlled studies. Behav Res Ther 1986, 25:397-409.

22. Cotler SB: Sex differences and generalization of anxiety reduction with automated desensitization and minimal therapist interaction. Behav Res Ther 1970, 8:273-285.

23. Donner L, Guerney BG, Jr: Automated group desensitization for test anxiety. Behav Res Ther 1969, 7:1-13.

24. van Boeijen CA, van Balkom AJ, van Oppen P, et al.: Efficacy of self-help manuals for anxiety disorders in primary care: a systematic review. Fam Pract 2005, 22:192-196.

25. den Boer PC, Wiersma D, Van den Bosch RJ: Why is selfhelp neglected in the treatment of emotional disorders? A meta-analysis. Psychol Med 2004, 34:959-971.

26. Andersson G, Carlbring P, Holmstrom A, et al.: Internetbased self-help with therapist feedback and in vivo group exposure for social phobia: a randomized controlled trial. $J$ Consult Clin Psychol 2006, 74:677-686.

27. Carlbring P, Ekselius L, Andersson G: Treatment of panic disorder via the Internet: a randomized trial of CBT vs. applied relaxation. J Behav Ther Exp Psychiatry 2003, 34:129-140.

28. Klein B, Richards JC, Austin DW: Efficacy of Internet therapy for panic disorder. J Behav Ther Exp Psychiatry 2006, 37:213-238.

29. Hirai M, Clum GA: An Internet-based self-change program for traumatic event related fear, distress, and maladaptive coping. J Trauma Stress 2005, 18:631-636. 
30. Carlbring P, Nilsson-Ihrfelt E, Waara J, et al.: Treatment of panic disorder: live therapy vs. self-help via the Internet. Behav Res Ther 2005, 43:1321-1333.

31. Lange A, Rietdijk D, Hudcovicova M, et al.: Interapy: a controlled randomized trial of the standardized treatment of posttraumatic stress through the internet. J Consult Clin Psychol 2003, 71:901-909.

32. Ritterband LM, Andersson G, Christensen HM, et al.: Directions for the International Society for Research on Internet Interventions (ISRII). J Med Internet Res 2006, 8:e23.

33. Spek V, Cuijpers P, Nyklicek I, et al.: Internet-based cognitive behaviour therapy for symptoms of depression and anxiety: a meta-analysis. Psychol Med 2007, 37:319-328.

The first published meta-analysis on Internet-based cognitivebehavioral therapy for depression and anxiety showing promising results for this type of intervention and marking the importance of proper guidance in Internet-based self-help interventions.

34. Mataix-Cols D, Marks IM: Self-help with minimal therapist contact for obsessive-compulsive disorder: a review. Eur Psychiatry 2006, 21:75-80.

35. van 't Veer-Tazelaar N, van Marwijk H, van Oppen P, et al.: Prevention of anxiety and depression in the age group of 75 years and over: a randomised controlled trial testing the feasibility and effectiveness of a generic stepped care programme among elderly community residents at high risk of developing anxiety and depression versus usual care [ISRCTN26474556]. BMC Public Health 2006, 6:186.

36. Newman MG: Recommendations for a cost-offset model of psychotherapy allocation using generalized anxiety disorder as an example. J Consult Clin Psychol 2000, 68:549-555.

37. Wang PS, Lane M, Olfson M, et al.: Twelve-month use of mental health services in the United States: results from the National Comorbidity Survey Replication. Arch Gen Psychiatry 2005, 62:629-640.
38. Goldberg D, Huxley P: Common Mental Disorders: A Biosocial Model. London: Tavistock/Routledge; 1992.

39. Andersson G: Internet-based cognitive-behavioral self help for depression. Expert Rev Neurother 2006, 6:1637-1642.

40. Andersson G, Bergstrom J, Carlbring P, Lindefors N: The use of the Internet in the treatment of anxiety disorders. Curr Opin Psychiatry 2005, 18:73-77.

41. Schneider AJ, Mataix-Cols D, Marks IM, Bachofen M: Internet-guided self-help with or without exposure therapy for phobic and panic disorders. Psychother Psychosom 2005, 74:154-164.

42. Kenwright M, Marks IM: Computer-aided self-help for phobia/panic via Internet at home: a pilot study. $\mathrm{Br} J$ Psychiatry 2004, 184:448-449.

43. Gega L, Norman IJ, Marks IM: Computer-aided vs. tutordelivered teaching of exposure therapy for phobia/panic: randomized controlled trial with pre-registration nursing students. Int J Nurs Stud 2007, 44:397-405.

44. Rosen GM, Glasgow RE, Barrera M, Jr: A controlled study to assess the clinical efficacy of totally self-administered systematic desensitization. J Consult Clin Psychol 1976, 44:208-217.

45. Scogin FR, Hanson A, Welsh D: Self-administered treatment in stepped-care models of depression treatment. J Clin Psychol 2003, 59:341-349.

46. Sobell MB, Sobell LC: Stepped care as a heuristic approach to the treatment of alcohol problems. J Consult Clin Psychol 2000, 68:573-579.

47. Newman MG, Erickson T, Przeworski A, Dzus E: Self-help and minimal-contact therapies for anxiety disorders: is human contact necessary for therapeutic efficacy? J Clin Psychol 2003, 59:251-274. 